\title{
Combining wearable sensor signals, machine learning and biomechanics to estimate tibial bone force and damage during running
}

\author{
Emily S. Matijevich ${ }^{1}$, Leon R. Scott ${ }^{2}$, Peter Volgyesi ${ }^{3}$, Kendall H. Derry $^{4}$ and Karl E. Zelik ${ }^{1,4,5}$ \\ ${ }^{1}$ Department of Mechanical Engineering, Vanderbilt University, Nashville, TN, USA, ${ }^{2}$ Department of \\ Orthopaedics, Vanderbilt University, Nashville, TN, USA, ${ }^{3}$ Institute for Software Integrated Systems, Vanderbilt \\ University, Nashville, TN, USA, ${ }^{4}$ Department of Biomedical Engineering, Vanderbilt University, Nashville, TN, \\ USA, ${ }^{5}$ Department of Physical Medicine \& Rehabilitation, Vanderbilt University, Nashville, TN, USA
}

Received: 14 October 2020

For correspondence: emily.matijevich@vanderbilt.edu

Please cite as: Matijevich, E., Scott, L., Volgyesi, P., Derry, K., \& Zelik, E. (2020). Achieving drastically improved estimates of tibial bone force and damage during running using wearable sensors. SportR $\chi i v$.

https://doi.org/10.31236/osf.io/vesh3

\begin{abstract}
There are tremendous opportunities to advance science, clinical care, sports performance, and societal health if we are able to develop tools for monitoring musculoskeletal loading (e.g., forces on bones or muscles) outside the lab. While wearable sensors enable non-invasive monitoring of human movement in applied situations, current commercial wearables do not estimate tissue-level loading on structures inside the body. Here we explore the feasibility of using wearable sensors to estimate tibial bone force during running. First, we used lab-based data and musculoskeletal modeling to estimate tibial force for ten participants running across a range of speeds and slopes. Next, we converted lab-based data to signals feasibly measured with wearables (inertial measurement units on the foot and shank, and pressure-sensing insoles) and used these data to develop two multi-sensor algorithms for estimating peak tibial force: one physics-based and one machine learning. Additionally, to reflect current running wearables that utilize running impact metrics to infer musculoskeletal loading or injury risk, we estimated tibial force using a commonly measured impact metric, the ground reaction force vertical average loading rate (VALR). Using VALR to estimate peak tibial force resulted in a mean absolute percent error of 9.9\%, which was no more accurate than a theoretical step counter that assumed the same peak force for every
\end{abstract}

All authors have read and approved this version of the manuscript.

This article was last modified on January 6, 2020.
Authors Emily Matijevich @EmilyMatijevich and Karl Zelik @KarlZelik can be reached on Twitter. 
running stride. Our physics-based algorithm reduced error to 5.2\%, and our machine learning algorithm reduced error to $2.6 \%$. Further, to gain insights into how force estimation accuracy relates to overuse injury risk, we computed bone damage expected due to a given loading cycle. We found that modest errors in tibial force translated into large errors in bone damage estimates. For example, a 9.9\% error in tibial force using VALR translated into $104 \%$ error in estimated bone damage. Encouragingly, the physics-based and machine learning algorithms reduced damage errors to $41 \%$ and $18 \%$, respectively. This study highlights the exciting potential to combine wearables, musculoskeletal biomechanics and machine learning to develop more accurate tools for monitoring musculoskeletal loading in applied situations.

\section{Introduction}

Monitoring loads on musculoskeletal structures inside the body is challenging in the laboratory, and remains an unsolved grand challenge in applied situations outside the lab. Inside the lab, musculoskeletal loads (e.g., forces, moments, stresses, strains) are typically estimated in one of two ways: (i) using implantable or percutaneous instrumentation to directly monitor tissue stress or strain (e.g., Komi, 1990; Lanyon et al., 1975) or (ii) using non-invasive instrumentation such as motion capture and force plates along with musculoskeletal modeling to indirectly estimate tissue loads (e.g., Burdett, 1982; Sasimontonkul et al., 2007; Scott \& Winter, 1990). While these two approaches have fueled decades of musculoskeletal biomechanics research, they can be impractically invasive and/or require sophisticated instrumentation and expertise. Thus, these approaches are typically limited to small sample sizes and infrequent data collections, which severely restricts collection of movement data in applied situations (i.e., real-world or daily activities).

There are many practical applications if non-invasive monitoring of musculoskeletal loads was possible in applied situations (Halilaj et al., 2018; Lim \& D'Souza, 2020; Patel et al., 2012; Peake et al., 2018; Ranavolo et al., 2018; Wang \& Adamczyk, 2019; Willy, 2018). Musculoskeletal load monitoring in combination with other information could provide transformative new health monitoring tools. For instance, an injury risk monitoring tool that monitors musculoskeletal loading in combination with other risk factors could help athletes coordinate training and rest days accordingly. Similarly, a clinical tool that monitors musculoskeletal loading in combination with other patient specific physiological measures (e.g., tissue quality, diet, menstrual cycles, sleep/rest information) could help researchers investigating overuse injury etiology. A biofeedback tool that combines musculoskeletal monitoring and specified thresholds or targets could help clinicians, trainers or ergonomists deliver objective biofeedback training to help ensure safe and efficient return to play or return to work after an injury. Finally, a solely musculoskeletal load monitoring tool could enable researchers, assistive device developers, or footwear/sports accessory designers to evaluate the effect of interventions on specific musculoskeletal loads outside the lab and over much longer time scales than is currently practical.

Small, portable, inexpensive wearable sensors along with accompanying software algorithms and user interfaces (together termed wearables) provide exciting opportunities for non-invasive monitoring of human movement in applied situations without the need for sophisticated lab equipment. Wearables often contain sensors such as inertial measurement units (IMUs), global positioning systems (GPS), or pressure or strain sensors. These wearables typically track spatiotemporal metrics (such as cadence, speed, number 
of steps taken, or time spent being active), body segment motions (such as accelerations or orientations), or interaction forces between the person and the environment (such as insole pressures or ground reaction forces, GRF). These metrics, particularly GRF metrics, are then often used to evaluate injury risk to structures inside the body. However, GRF metrics are generally not indicative of loads experienced by musculoskeletal structures in the body (e.g., tibial force), nor is there consistent epidemiological evidence to support the use of GRF metrics as indicators of bone stress injury (Matijevich et al., 2019). See Matijevich et al., 2019 and Vigotsky et al., 2019 for extended discussions on the widespread misuse of GRF metrics to infer internal tissue loading and associated injury risk in both scientific research and commercial wearables. To date there are only a very limited number of wearables (mostly noncommercial, in-development) that have demonstrated validity or reported accuracy in estimating the mechanical loading on specific structures inside the body (e.g., Martin et al., 2018).

There are numerous knowledge gaps surrounding how to more accurately estimate musculoskeletal loads with wearables. Many inter-related choices must be made in order to develop a new wearable, for instance: What type(s) of wearable sensor(s) to use? What algorithm(s) to employ to fuse multi-sensor data? Which musculoskeletal loading metrics to track, and what level of accuracy is desired? There are innumerable approaches for tackling the many intertwined and open-ended questions, making this an interesting opportunity for exploratory research that combines wearables, musculoskeletal biomechanics and machine learning. To manageably explore these choices, we targeted a specific musculoskeletal structure and movement: the tibial (shin) bone during running. We were motivated to monitor tibial force because tibial overuse injuries (e.g., bone stress fractures) are a common debilitating injury amongst runners, athletes and military cadets (Bennell \& Brukner, 1997; Brukner et al., 1996; Cosman et al., 2013; Hulkko \& Orava, 1987; Matheson et al., 1987; Yagi et al., 2013); however, we believe the risk of injury can be reduced if tibial forces can be monitored in daily life. Further, there are established and validated methodologies for non-invasively estimating tibial compression force using labbased instrumentation and musculoskeletal modeling (Burdett, 1982; Sasimontonkul et al., 2007; Scott \& Winter, 1990), giving us confidence in our target bone force metric.

The objective of this study was to develop multi-sensor algorithms for estimating tibial force using idealized wearable sensor signals, quantify the accuracy of each algorithm, and then compare the accuracy of these algorithms to approaches that rely on ground reaction force metrics or other single variable surrogates to try to gain insight on internal loading or injury risk. Here we summarize the development of two promising multi-sensor algorithms (one physics-based and one using machine learning) for estimating peak tibial force across various running speeds and slopes. For reference, we also report two other algorithms (single variable linear regression and group mean), which were intended to reflect the current state-of-the-art for commercial wearables.

\section{Methods}

\section{Data collection}

In this study we re-analyzed an existing dataset from our prior publication (Matijevich et al., 2019), in which ten recreational runners performed 30 running conditions where for each condition the running speed and slope was varied. These data are publicly archived (Matijevich et al., 2019). The 
dataset includes 5 males and 5 females (age: $24 \pm 2.5$ years; height: $1.7 \pm 0.1 \mathrm{~m}$; mass: $67 \pm 6 \mathrm{~kg}$ ) all of whom reported running a minimum of 10 miles per week. All participants gave written informed consent to the original protocol, which was approved by the Institutional Review Board at Vanderbilt University. To briefly summarize methods, 30-s trials were collected across a range of speeds ( 2.6 to $4.0 \mathrm{~m} / \mathrm{s}$ ) and slopes ( -9 to +9 degrees), while runners wore their own personal running shoes. Ground reaction forces were collected at $1000 \mathrm{~Hz}$ on a force-instrumented treadmill (Bertec), synchronously with unilateral lower-limb kinematics at $100 \mathrm{~Hz}$ (Vicon). For further details on the experimental protocol or rationale underlying the running conditions see Matijevich et al., 2019.

\section{Lab-based data analysis}

Data were analyzed to estimate tibial compression force as detailed in (Matijevich et al., 2019), thus only briefly summarized below. Lower-body segmental kinematics were estimated based on optical motion capture data and rigid-body inverse kinematics. GRF and kinematics were combined via rigidbody inverse dynamics to estimate joint kinetics (C-Motion, Visual3D). The time-series compression force acting on the distal end of the tibia during the stance phase of running was then estimated using a simple musculoskeletal model (Matijevich et al., 2019); by summing estimates of the net force on the ankle and the compression force from plantarflexor calf muscles (Fig. 1). We chose peak tibial force of each running condition as our target metric because maximum force is a key factor that contributes to cyclic fatigue of bone and resulting damage accumulation (see Matijevich et al., 2019 for additional discussion). Peak tibial force was estimated as the maximum force across stance on a step-by-step basis, and then averaged to compute the mean for each condition $\left(F_{\max }\right)$, reported in units of body weight $(\mathrm{BW})$.

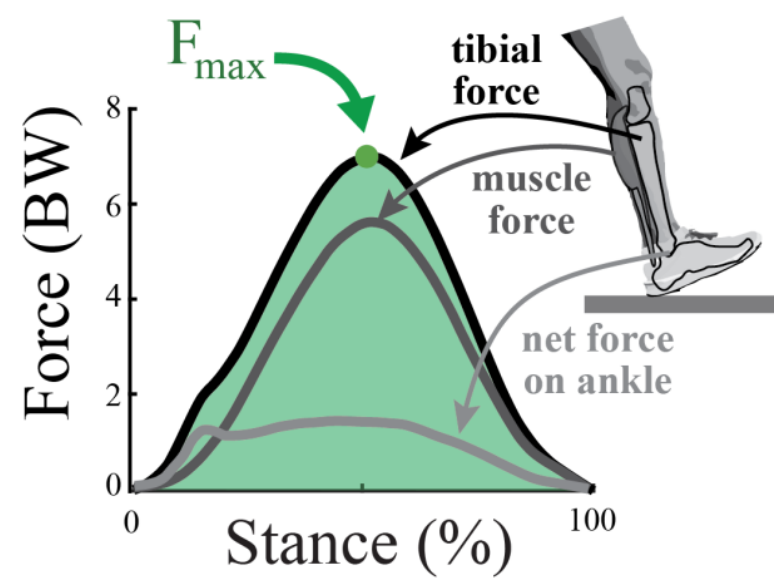

Figure 1. Tibial compression force was computed from motion capture and ground reaction force data using musculoskeletal modeling: by summing the net force on the ankle and the compression force from plantarflexor calf muscles. Peak tibial force $\left(F_{\text {max }}\right)$ in bodyweights $(\mathrm{BW})$ during stance was computed for each running condition. The lab-based estimate of $F_{\text {max }}$ is the target load metric that we are interested in monitoring with wearable sensors. 


\section{Candidate wearable sensor signal identification and data preparation}

Before developing and training algorithms to estimate our target load metric (peak tibial force, $\left.F_{\max }\right)$ we first identified candidate wearable sensor signals to use as algorithm inputs. Candidate wearable sensor signals were selected because they could feasibly be collected with existing commercial wearable sensors (e.g., pressure-sensing insoles, IMUs) and we expected them to be important to the estimation of tibial force based on mechanics (e.g., signals typically used in inverse dynamics and musculoskeletal models of the lower limb). The four candidate sensor signals we selected were foot and shank orientations, vertical GRF and center of pressure (CoP, the point of application of the GRF vector under the foot).

We considered two options for using wearable sensor signals to develop tibial force estimation algorithms: (i) performing new experiments where we collect data from multiple wearable sensors synchronously with lab-based instrumentation, or (ii) converting lab-based data into the types of signals that could be feasibly obtained with existing commercial wearables (e.g., simplifying the three dimensional GRF vector into a one dimensional normal force, which represents the type of signal that can be estimated from a pressure-sensing insole). We chose the second option and termed these signals idealized wearable signals. Idealized wearable signals were beneficial for this feasibility study because they allowed us to not be limited by the quality or accuracy of existing wearables. In reality, sensor hardware, algorithms and signal quality are constantly improving; for example, sophisticated filtering has improved the quality of absolute angle estimates from IMUs (Picerno, 2017). Further, idealized wearable signals allowed us to explore a broader combination of sensor signals from our pre-existing dataset, and eliminated the need to select and integrate a specific sensor set and carry out new experiments before knowing which subset of sensors were actually needed.

In Table 1, we summarize how we converted lab-based data into idealized wearable signals. Algorithms used either time-series idealized wearable signals as inputs or discrete features extracted from these signals as inputs. Discrete features were computed as follows: Vertical GRF features - vertical average loading rate (VALR), active peak, impulse - were extracted using established methodologies as detailed in Matijevich et al., 2019. Foot/shank minimum and maximum angles were the minimum and maximum sagittal plane angles during the stance phase of running. Foot/shank angles at midstance were the sagittal plane angles at the time of the vertical GRF active peak. CoP at midstance was the mediallateral and anterior-posterior position of the $\mathrm{CoP}$ at the time of vertical GRF active peak. Stance time was the time from foot contact to toe off. Stride time was the time from foot contact to the next ipsilateral foot contact. All idealized wearable signals and discrete features were computed on a step-by-step basis, and then averaged to compute the mean for each running condition. Condition means were used in algorithm development and evaluation, and were computed from approximately 25-30 strides for each condition. 
Table 1. Idealized wearable signals. Summary of our selected idealized wearable signals, existing wearable sensors that can estimate these types of signals, how lab-based data were converted into idealized wearable signals, and which discrete features were extracted from these signals.

\begin{tabular}{|c|c|c|c|}
\hline $\begin{array}{l}\text { Idealized } \\
\text { wearable } \\
\text { signal }\end{array}$ & $\begin{array}{l}\text { Wearable sensors } \\
\text { examples of existing } \\
\text { sensor hardware that can } \\
\text { be used to estimate each } \\
\text { signal }\end{array}$ & $\begin{array}{l}\text { Data conversion } \\
\text { how lab-based signals } \\
\text { were converted into } \\
\text { idealized wearable } \\
\text { signals }\end{array}$ & $\begin{array}{l}\text { Discrete features } \\
\text { features extracted from } \\
\text { the idealized wearable } \\
\text { signals }\end{array}$ \\
\hline $\begin{array}{l}\text { Shank angle } \\
\text { (sagittal) }\end{array}$ & IMU on the shank & $\begin{array}{l}\text { Inverse kinematics } \\
\text { processed to output shank } \\
\text { orientation in the lab } \\
\text { frame }\end{array}$ & $\begin{array}{l}\text { Min angle, max angle, } \\
\text { angle at midstance }\end{array}$ \\
\hline $\begin{array}{l}\text { Foot angle } \\
\text { (sagittal) }\end{array}$ & IMU on the foot/shoe & $\begin{array}{l}\text { Inverse kinematics } \\
\text { processed to output foot } \\
\text { orientation in the lab } \\
\text { frame }\end{array}$ & $\begin{array}{l}\text { Min angle, max angle, } \\
\text { angle at midstance }\end{array}$ \\
\hline Vertical GRF & Pressure-sensing insole & $\begin{array}{l}\text { 3D GRF }{ }^{\dagger} \text { transformed } \\
\text { into foot's coordinate } \\
\text { frame \& projected into } \\
1 \mathrm{D} \text { GRF normal to the } \\
\text { ground }\end{array}$ & $\begin{array}{l}\text { VALR, active peak, } \\
\text { impulse, stance time, } \\
\text { stride time }\end{array}$ \\
\hline $\mathrm{CoP}$ & Pressure-sensing insole & $\begin{array}{l}\text { GRF and motion capture } \\
\text { used to transform } \\
\text { treadmill CoP into the } \\
\text { foot's coordinate frame }\end{array}$ & $\begin{array}{l}\text { Medial-lateral \& } \\
\text { anterior-posterior CoP at } \\
\text { midstance }\end{array}$ \\
\hline Speed & IMU or GPS & Used treadmill speed & Mean running speed \\
\hline Slope & IMU & Used treadmill slope & Ground slope \\
\hline
\end{tabular}

Prior to extracting GRF metrics, the data were low-pass filtered at $45 \mathrm{~Hz}\left(3^{\text {rd }}\right.$ order, zero-lag

Butterworth) to avoid filtering out foot impact and loading rate dynamics.

\section{Algorithm development}

We developed two multi-sensor algorithms for more accurately estimating peak tibial force using idealized wearable sensor signals: one physics-based and one using machine learning. Additionally, we report two other algorithms: single variable linear regression and group mean. These were intended to reflect the current state-of-the-art for commercial wearables, which typically rely on VALR (or correlated metrics like impact peaks and lower limb peak accelerations) and/or step counters to provide musculoskeletal loading and injury risk feedback. See Matijevich et al., 2019 for an extended discussion (and specific examples) on the widespread misuse of GRF metrics to signify bone loading and associated injury risk in both scientific research and commercial wearables. For each algorithm, one or more of the idealized wearable signals or discrete features were used as inputs, and the output was an estimate of peak tibial force (in units of BW). To develop each algorithm, we used k-fold validation by runner, a 
commonly-used technique to assess the generalizability of an algorithm (Halilaj et al., 2018). In other words, we used data from nine runners to train the model (i.e., optimize model parameters), and then tested the model on the remaining runner. This train-test process was repeated for all ten combinations. Summary statistics were then calculated on accuracy. Below we first overview the algorithms themselves, followed by a description of how algorithm estimates were evaluated vs. lab-based estimates of tibial force. An overview of the lab-based data analysis and algorithm evaluation is provided in Fig. 2.

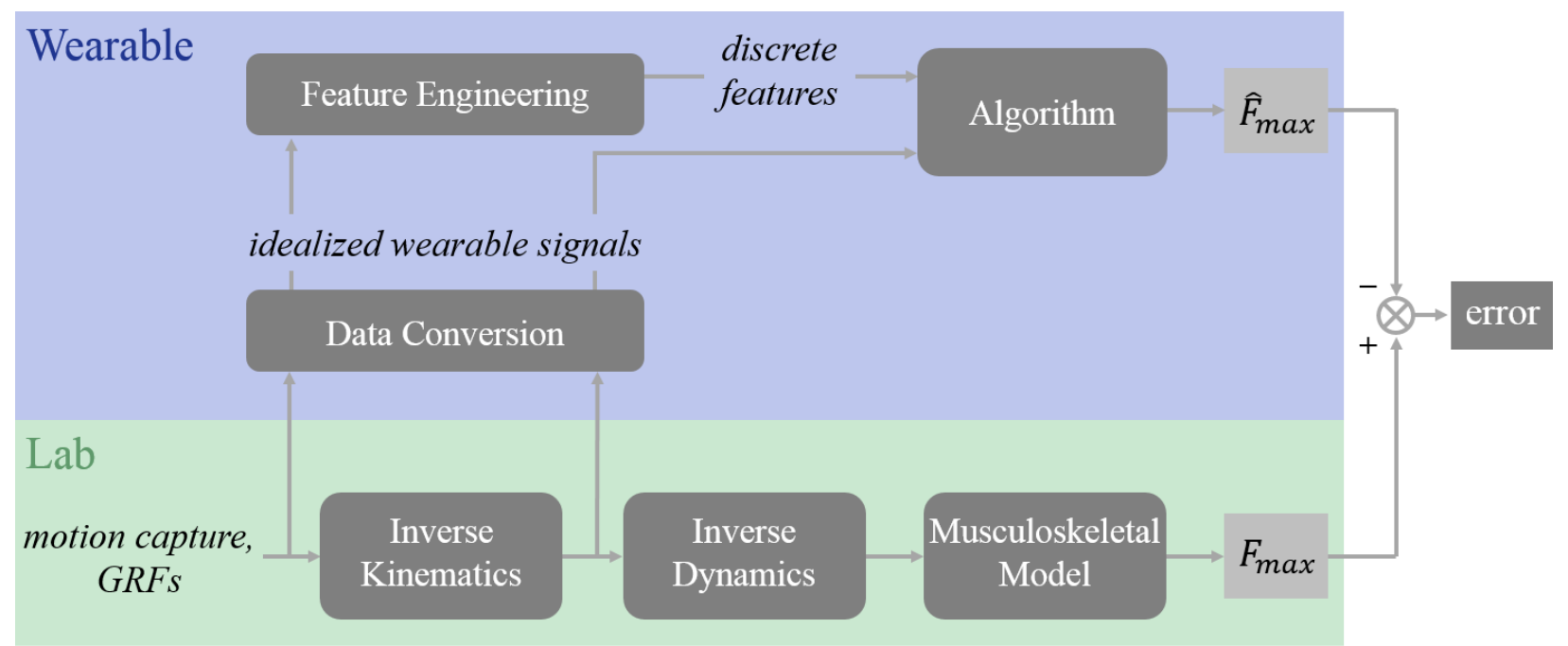

Figure 2. Lab-based data analysis and algorithm evaluation overview. (Lower, Lab) Lab-based data were collected (motion capture and GRFs) and standard inverse kinematics and inverse dynamics analyses were performed. A musculoskeletal model was used to calculate peak tibial force $\left(F_{\text {max }}\right)$, which was treated as ground truth bone load for the purposes of this study. (Upper, Wearable) Lab-based signals were converted to idealized wearable signals, representing the types of signals that can be obtained with existing commercial wearable sensors. Algorithms used idealized wearable signals and discrete features that were extracted from these signals as inputs. Algorithms output estimates of peak tibial force $\left(\hat{F}_{\text {max }}\right)$. Errors between the algorithm estimates and lab-based estimates of peak tibial force were computed.

\section{Single variable linear regression}

To reflect the current state-of-the-art in commercial wearables, we report an estimate of peak tibial force $\left(\hat{F}_{\text {max }}\right)$ based on the common assumption/misconception that VALR can serve as a surrogate for tibial force and/or injury risk (Worp et al., 2016; Zadpoor \& Nikooyan, 2011). VALR and lab-based estimates of peak tibial force $\left(F_{\max }\right)$ were used to find linear scaling coefficients by k-fold validation to estimate peak tibial force $\left(\widehat{F}_{\text {max }}\right)$. We selected VALR, in part, because unlike impact peak, it can be computed for every step regardless of the presence of a transient peak in the GRF (Matijevich et al., 2019). Based on our own curiosity, we also generated additional single variable linear regression models using other commonly tracked metrics: GRF active peak, GRF impulse, running speed and ground slope. 


\section{Group mean approach}

As another point of reference, we assumed that peak tibial force was constant for every step, regardless of speed or slope. Practically speaking, this approach represents what we would expect if we tried to use only a step counter (pedometer) to monitor bone injury risk. With this naïve modeling approach, we assumed that peak tibial force $\left(\widehat{F}_{\max }\right)$ for all conditions for a given runner was equal to the mean peak tibial force across the remaining runners and conditions.

\section{Physics-based algorithm}

We implemented a physics-based algorithm (Appendix A) using four idealized wearable sensor signals (Table 1). This algorithm is similar to how we estimate musculoskeletal forces in the laboratory using inverse dynamics and biomechanical modeling. However, the key difference is that wearable sensors only provide limited signals (e.g., one dimensional normal GRF rather than three-dimensional GRF), which are insufficient to compute the full equations of motion. There are innumerable ways to deal with incomplete data, including simplifying the equations of motion themselves (e.g., removing terms that are expected to have small effects or terms that are impractical to collect with wearables), or using available data to estimate the missing terms (e.g., using regression equations to approximate the complete 3D GRF vector using data from multiple insole pressure sensors (Fong et al., 2008; Jacobs \& Ferris, 2015)). Here, we used the former approach to provide a physics-based estimate of peak tibial force.

\section{Machine learning algorithm}

For the machine learning algorithm, we used 13 discrete features from our idealized wearable sensor signals to estimate peak tibial force (Table 1, discrete features). This set of discrete features was based on preliminary exploration of data - by analyzing time series signals, scatter plots and correlation values. Feature pruning was necessary to avoid overfitting the model with a limited dataset (Halilaj et al., 2018) and to speed up the hyperparameter search process. Features were normalized to z-scores prior to model training. We explored a variety of supervised machine learning techniques for multi-variable regression, including generalized linear models, ensemble methods, neural networks and support vector regression. Ultimately, we selected LASSO (Least Absolute Shrinkage and Selection Operator) regression, a least squares linear model with a $\mathrm{L}_{1}$ regularization. This regularization penalizes the sum of the absolute values of the coefficients and forces a subset of learned coefficient weights to zero, effectively selecting a sparser subset of features. In addition to providing estimates of peak tibial force $\left(\widehat{F}_{\text {max }}\right)$, the resulting feature weights are used to rank the relative importance of each discrete feature.

\section{Algorithm evaluation}

To evaluate each algorithm, we computed the mean absolute percent error (MAPE) between $F_{\text {max }}$ and $\widehat{F}_{\text {max }}$ across all conditions for each runner, and report the inter-runner mean, standard deviation, and range. In some instances, we also computed root mean square error (RMSE) in units of BW for reference. 


\section{Results}

MAPE results for the single variable linear regression and multi-sensor algorithms are shown in Fig. 3 and Table 2. Results in RMSE for reference: The single variable linear regression algorithm that used VALR to estimate peak tibial force $\left(\hat{F}_{\text {max }}\right)$ resulted in a RMSE of $0.97 \pm 0.32$ BWs (Fig. 3A, Table 2). The physics-based algorithm resulted in a RMSE of $0.48 \pm 0.09$ BWs (Fig. 3B, Table 2). The multivariable machine learning algorithm resulted in a RMSE of $0.25 \pm 0.07$ BWs (Fig. 3C, Table 2).
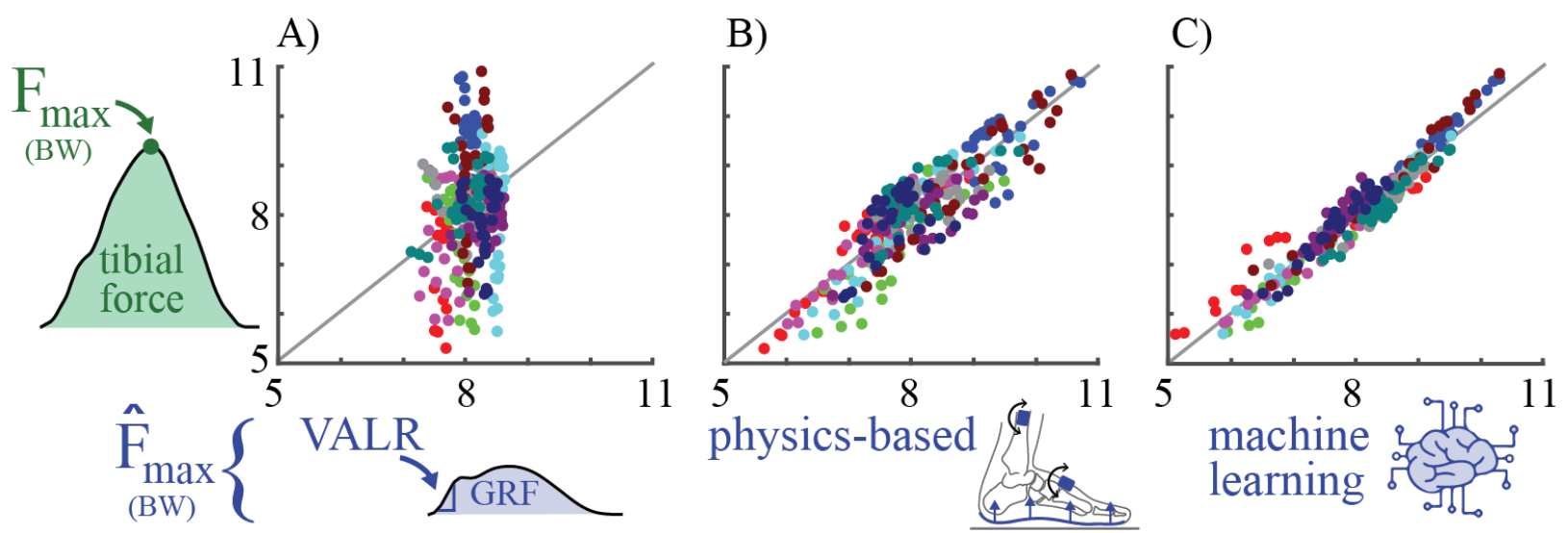

Figure 3. Comparison of force estimation algorithms. The plots depict the lab-based estimate of peak tibial force $\left(F_{\text {max }}\right)$ vs. wearable sensor algorithm estimates of peak tibial force $\left(\widehat{F}_{\text {max }}\right)$, in units of BW. Estimates are from A) single variable linear regression using VALR, B) physics-based algorithm, and C) machine learning algorithm. Colors represent 10 runners. Each point represents a single running speedslope combination (30 conditions per runner). A line with a slope of one is added to visualize a perfect correspondence between lab-based and wearable estimates.

Table 2. Average errors of force estimation algorithms. This table reports MAPE for algorithm estimates of peak tibial force $\left(\widehat{F}_{\text {max }}\right)$ relative to lab-based estimates of peak tibial force $\left(F_{\text {max }}\right)$. Shown are the inter-runner means, standard deviations and ranges $(N=10)$. The physics-based algorithm and machine learning algorithm exhibited lower force estimation errors than single variable linear regression algorithm using VALR ( $\mathrm{p}=0.004$ and $\mathrm{p}<0.001$ respectively, based on Wilcoxon signed-rank test on the k-fold crossvalidation error results).

\begin{tabular}{|c|c|c|c|}
\cline { 2 - 4 } \multicolumn{1}{c|}{} & $\begin{array}{c}\text { Single variable linear } \\
\text { regression }(V A L R)\end{array}$ & Physics-based & Machine learning \\
\hline mean \pm std & $9.9 \pm 3.5 \%$ & $5.2 \pm 1.3 \%$ & $2.6 \pm 0.8 \%$ \\
\hline$[\min \max ]$ & {$[4.5 \% 15.0 \%]$} & {$[3.8 \% 8.1 \%]$} & {$[1.5 \% 4.4 \%]$} \\
\hline
\end{tabular}

Other single variable linear regression estimates resulted in average MAPE of about 8-11\% (Fig. 4 and Table 3). Results in RMSE for reference: Using GRF active peak resulted in a RMSE of $0.77 \pm$ 0.12 BWs (Fig. 4A, Table 3). Using GRF impulse resulted in a RMSE of $1.03 \pm 0.38$ BWs (Fig. 4B, Table 3). Using ground slope resulted in a RMSE of $0.79 \pm 0.33$ BWs (Fig. 4C, Table 3). Using running speed resulted in a RMSE of $0.91 \pm 0.38$ BWs (Fig. 4D, Table 3). Using the group mean algorithm 
approach (i.e., assuming peak tibial force for a given runner was the same for every running condition) resulted in a RMSE of $0.99 \pm 0.38$ BWs (Fig. 4E, Table 3).
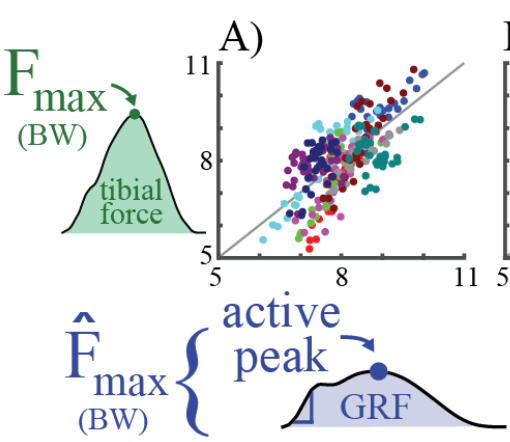

B)

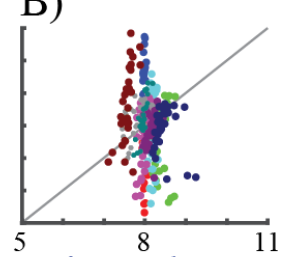

impulse

Figure 4. Comparison of additional single variable linear regression force estimation algorithms, and the group mean approach. The plots depict the lab-based estimate of peak tibial force $\left(F_{\text {max }}\right)$ vs. single variable linear regression estimates of peak tibial force $\left(\hat{F}_{\text {max }}\right)$, in units of BW. Single variable linear regression results used A) vertical GRF active peak, B) vertical GRF impulse, C) ground slope, and D) running speed. Peak tibial force estimates using E) the group mean force (i.e., a constant peak tibial force) are also plotted for reference. Colors represent 10 runners. Each point represents a single running speed-slope combination (30 conditions per runner). A line with a slope of one is added to visualize a perfect correspondence between lab-based and wearable estimates.

Table 3. Average errors of additional single variable linear regression force estimation algorithms, and the group mean approach. The table reports MAPE for algorithm estimates of peak tibial force $\left(\hat{F}_{\text {max }}\right)$ relative to lab-based estimates of peak tibial force $\left(F_{\text {max }}\right)$. Shown are the inter-runner means, standard deviations and $\min / \max$ ranges $(N=10)$.

\begin{tabular}{|c|c|c|c|c|c|}
\cline { 2 - 5 } \multicolumn{1}{c|}{} & \multicolumn{4}{c|}{ Single variable linear regression } & \multirow{2}{*}{$\begin{array}{c}\text { Group mean } \\
\text { force }\end{array}$} \\
\cline { 2 - 6 } \multicolumn{1}{c|}{} & $\begin{array}{c}\text { Vertical GRF } \\
\text { active peak }\end{array}$ & $\begin{array}{c}\text { Vertical GRF } \\
\text { impulse }\end{array}$ & Slope & Speed & \\
\hline mean \pm std & $8.2 \pm 1.6 \%$ & $10.5 \pm 3.9 \%$ & $8.5 \pm 3.4 \%$ & $9.6 \pm 4.2 \%$ & $10.3 \pm 4.0 \%$ \\
\hline$[$ min max $]$ & {$[5.6 \% 10.4 \%]$} & {$[5.3 \% 15.2 \%]$} & {$[4.0 \% 16.1 \%]$} & {$[4.1 \% 15.9 \%]$} & {$[5.1 \% 15.8 \%]$} \\
\hline
\end{tabular}

\section{Discussion}

Here we demonstrate two multi-sensor algorithms that provide improved estimates of peak tibial force during running across speeds and slopes, as compared to conventional approaches used by current wearables. On average, the physics-based algorithm had 5\% error and the machine learning algorithm has a 3\% error, compared to the single variable linear regression approach using VALR or other GRF and single variable metrics that had average errors ranging from $8-11 \%$. These findings highlight that multisensor algorithms offer a promising and feasible approach for more accurately estimating musculoskeletal loads, like tibial force, using wearable sensors in applied situations. 


\section{Encouraging implications for using existing wearable sensors with improved algorithms}

Fortunately, all the sensor signals and discrete features used in these algorithms can be obtained from existing wearable sensor hardware. This suggests that there are opportunities to achieve more accurate musculoskeletal load estimates solely by selecting appropriate sensors and accompanying software algorithms. Specifically, the physics-based algorithm used time series shank angle, foot angle, GRF magnitude, and anterior-posterior CoP under the foot, which could be obtained with IMUs placed on the shoe and shank and a pressure-sensing insole/shoe. For the machine learning algorithm, all discrete features could be estimated from signals obtained from the same IMUs and pressure-sensing insole/shoe.

While our reported machine learning algorithm used discrete features, which could be obtained from three wearable sensors, we also explored how reducing the number of sensors used in the algorithm to just one or two would influence algorithm estimates (Table 4). Three interesting insights we observed were: (i) Adding the shank IMU features to the pressure insole and foot IMU features had minimal effect on the tibial force estimation error. This was corroborated by outputs from the LASSO algorithm as after training the original machine learning model, the shank features were all ranked as low importance. (ii) Relatedly, the models including both the pressure insole and foot IMU resulted in the lowest errors. This was also consistent with LASSO outputs, as the original model ranked three features from these sensors as the highest importance: GRF active peak, anterior-posterior CoP at midstance, and the foot angle at midstance. (iii) Each of these iterations used more than a single feature to estimate peak tibial force and three of the combinations resulted in an error less than our single variable linear regression estimates or group mean approach, and three of the combinations resulted in an error equal to our single variable linear regression estimates or group mean approach (Table 3 vs. Table 4). While it would be extremely convenient if a single metric/feature was a strong indicator of tibial force, we show that by combining just a few data features we are able to improve our peak tibial force estimates (average error of $<3 \%$ compared to $\sim 10 \%$ using a single metric). While not reported here, a similar exploration of resulting algorithm estimates using different sensor combinations could be repeated for the physics-based approach. 
Table 4. Errors in tibial force estimates for machine learning algorithms using different sensor combinations. The table reports MAPE of wearable algorithm estimates of peak tibial force $\left(\widehat{F}_{\text {max }}\right)$ compared to lab-based estimates of peak tibial force $\left(F_{\max }\right)$. Each iteration used a different combination of wearable sensors providing a different number of discrete features. Refer to Table 1 for an explanation of which discrete features were available from each idealized wearable sensor. Reported are the interrunner means and standard deviations $(N=10)$. Note: The first combination is equivalent to the subset used in our presented machine learning mode (equivalent to Fig. 3 and Table 2) and is included here for reference.

\begin{tabular}{|l|l|}
\hline $\begin{array}{l}\text { Wearable sensors used } \\
(\mathbf{f}=\text { total number of discrete features used })\end{array}$ & $\begin{array}{l}\text { MAPE mean } \pm \\
\text { std }(\mathbf{N = 1 0})\end{array}$ \\
\hline $\begin{array}{l}\text { pressure insole + foot IMU + shank IMU } \\
(\mathrm{f}=13)\end{array}$ & $2.6 \pm 0.8 \%$ \\
\hline pressure insole + foot IMU (f=10) & $2.6 \pm 0.6 \%$ \\
\hline pressure insole (f=7) & $4.7 \pm 1.7 \%$ \\
\hline foot IMU + shank IMU (f=9) & $8.3 \pm 4.9 \%$ \\
\hline shank IMU (f=5) & $8.0 \pm 2.9 \%$ \\
\hline foot IMU (f=5) & $7.9 \pm 2.3 \%$ \\
\hline
\end{tabular}

\section{Opportunities for advancement of wearables that monitor musculoskeletal loads}

There are numerous opportunities for further exploration and improvement of the two algorithms presented here. First, now that candidate sensors and signals/features have been identified in this study, a fully portable/wearable system could be developed and its accuracy could be assessed. It is important to remind that idealized wearable signals allowed us to identify the most promising signals and features; however, in many instances a given signal or feature could be estimated from multiple different types of sensors. For example, GRF active peak could be estimated either with a pressure insole, or with a waistmounted accelerometer (Kiernan et al., 2018; Neugebauer et al., 2014). Thus, there may be opportunities to strategically select sensor hardware to accommodate application constraints (e.g., a footwear developer may opt to measure GRF active peak with an insole, whereas an apparel developer may opt to integrate an accelerometer into the waistband of shorts).

Second, an additional algorithm approach is to fuse the physics-based and machine learning algorithms, each of which has its own benefits and challenges. The physics-based algorithm is based on equations of motion that describe the system (the runner's tibial bone), which theoretically should estimate force accurately beyond the running conditions tested here. However, as detailed in methods, current wearable sensors provide limited signals that are insufficient to compute the full equations of motion. The LASSO machine learning algorithm is advantageous because it uses a small set of idealized wearable sensor features and develops a model that minimizes tibial force estimation error in the training data. However, the final model is dependent on the training data and should not be assumed to extrapolate to running conditions beyond the training set. Potentially, one could first estimate tibial force using the physics-based algorithm and then use these results, in addition to other wearable features, as inputs to the machine learning model. This would leverage the strengths of each algorithm. Another interesting and yet 
unexplored research direction is to build and train non-linear neural network models with internal structures influenced by the physics-based equations - effectively embedding the domain knowledge (biomechanics) into the network architecture. For example, if from inverse dynamics we know that CoP will be multiplicatively related to GRF (to estimate ankle moment), we could add that non-linearity into the neural network model structure.

Third, as new sensor hardware is developed, it is expected to further enhance musculoskeletal load estimation accuracy. For instance, future commercial pressure insoles may be able to measure the anterior-posterior GRF, providing more complete input signals for physics-based algorithms, and additional candidate features for machine learning. Ultimately, the desired estimation accuracy will vary based on the intended application as there is some tradeoff between hardware complexity and estimation accuracy.

\section{Discouraging implications for VALR and other single variable surrogates for musculoskeletal load}

VALR was a poor predictor of tibial bone force. The average force errors from VALR $(9.9 \%$, $0.97 \mathrm{BWs})$ were nearly the same as using the naive group mean approach $(10.3 \%, 0.99 \mathrm{BWs})$, which simply assumes a constant peak tibial force for every step. These findings suggest that if our goal is to monitor peak force on the tibial bone, then tracking VALR may be no more accurate than simply using a pedometer (step counter) and assuming a constant mean force for every step. Of note, for the group mean approach presented here to emulate a step counter, we chose to use the group mean of peak tibial force, but any other average metrics (median, mode, etc.) would similarly result in estimates that do not capture trends in tibial force (Fig. 4E).

The machine learning algorithm results provide further evidence of the limited utility of VALR. VALR was the one and only discrete feature for which the LASSO machine learning algorithm drove the weighting factor to zero, indicating that VALR was not needed or used in model predictions. These findings may be surprising to some readers given the fact that VALR (or correlated signals like GRF impact peak, peak foot/shank accelerations (Crowell, 2010; Hamill et al., 1995; Hennig et al., 1993), etc.) is commonly assumed in sport science, biomechanics and related disciplines to signify athlete workload, internal loading or injury risk. However, as discussed in Matijevich et al., 2019 and Vigotsky et al., 2019, we believe these assumptions/interpretations reflect fundamental misconceptions in the field regarding the relationship between external forces (between the foot and the ground), the loads experienced inside the body by musculoskeletal structures (e.g. Fig. 3A), and the resulting damage and injury risk. Thus, we continue to urge vigilance in how VALR and other impact-related metrics are used and interpreted within the context of sport science and injury risk assessment.

\section{Important insights gained when considering tibial damage}

The methods above describe how we assessed the bone force estimation accuracy of each algorithm, which enables us to evaluate relative performance of algorithms. However, an open question in the research field is: what level of musculoskeletal force estimation accuracy is needed for assessing overuse injury risk? It is impossible to know definitively at this stage and deciding when a model is accurate enough is a known challenge in the field (see Halilaj et al., 2018 and the following Discussion 
section 4.5). Nevertheless, one way to gain insight is to consider the relationship between bone force and bone microdamage, since overuse injuries are believed to result from an accumulation of microdamage (Edwards, 2018). Here we use the word damage to refer to the mechanical fatigue-induced microstructural damage to bone tissue (Edwards, 2018).

There is extensive experimental evidence that material (e.g., bone) damage is exponentially related to the force applied (Damage $\alpha$ Force $^{7}$, Carter \& Caler, 1985; Currey, 2013; Edwards, 2018, see Appendix B for additional details). As an example of how even modest errors in force estimates result in enormous errors in bone damage estimates, $8-11 \%$ errors in tibial force estimates based on single variable linear regressions would translate, on average, to bone damage errors of about 70-120\% (Fig. B2 \& B3, Tables B1 \& B2). Alternatively, 5\% errors in tibial force estimated using the physics-based algorithm would translate to about $41 \%$ error in bone damage and $3 \%$ errors in tibial force estimated using the machine learning algorithm would translate to about $18 \%$ error in bone damage (Fig. B2, Table B1). See Appendix B for full bone damage results.

To gain intuition into how errors in our force and damage estimates may accrue over a series of loading cycles (i.e., over multiple running strides), we also estimated cumulative damage, using both our lab-based estimate of peak tibial force and our algorithm estimates of peak tibial force, over simulated five-mile running sessions. For brevity of the main text, these cumulative damage methods and full results are presented in Appendix B, with one finding presented here:

When comparing 25 simulated running workouts of differing intensity (varying number of running strides at different combinations of speeds and slopes), our lab-based estimates of cumulative damage resulted in different amounts of cumulative bone damage per run (Fig. 5). If the runner used the VALR linear regression based estimates of cumulative damage, they would be misguided about how much damage their tibial bone experiences during each workout, with some workouts being largely overestimated and some being largely underestimated. There are important consequences to severely over- or under-estimating damage to the bone. Large overestimates of bone damage may result in wearables that advise to sideline an athlete unnecessarily, while large underestimates of damage may result in a failure to notify an athlete of heightened risk before an injury occurs. Alternatively, estimating cumulative damage per run with the physics-based or machine learning algorithm more accurately estimates the bone damage per run (Fig. 5); capturing key trends in higher vs. lower damage workouts. These two multi-sensor algorithms offer an exciting and powerful tool for capturing important trends in bone damage and potentially overuse injury risk (due to microdamage accumulation). 


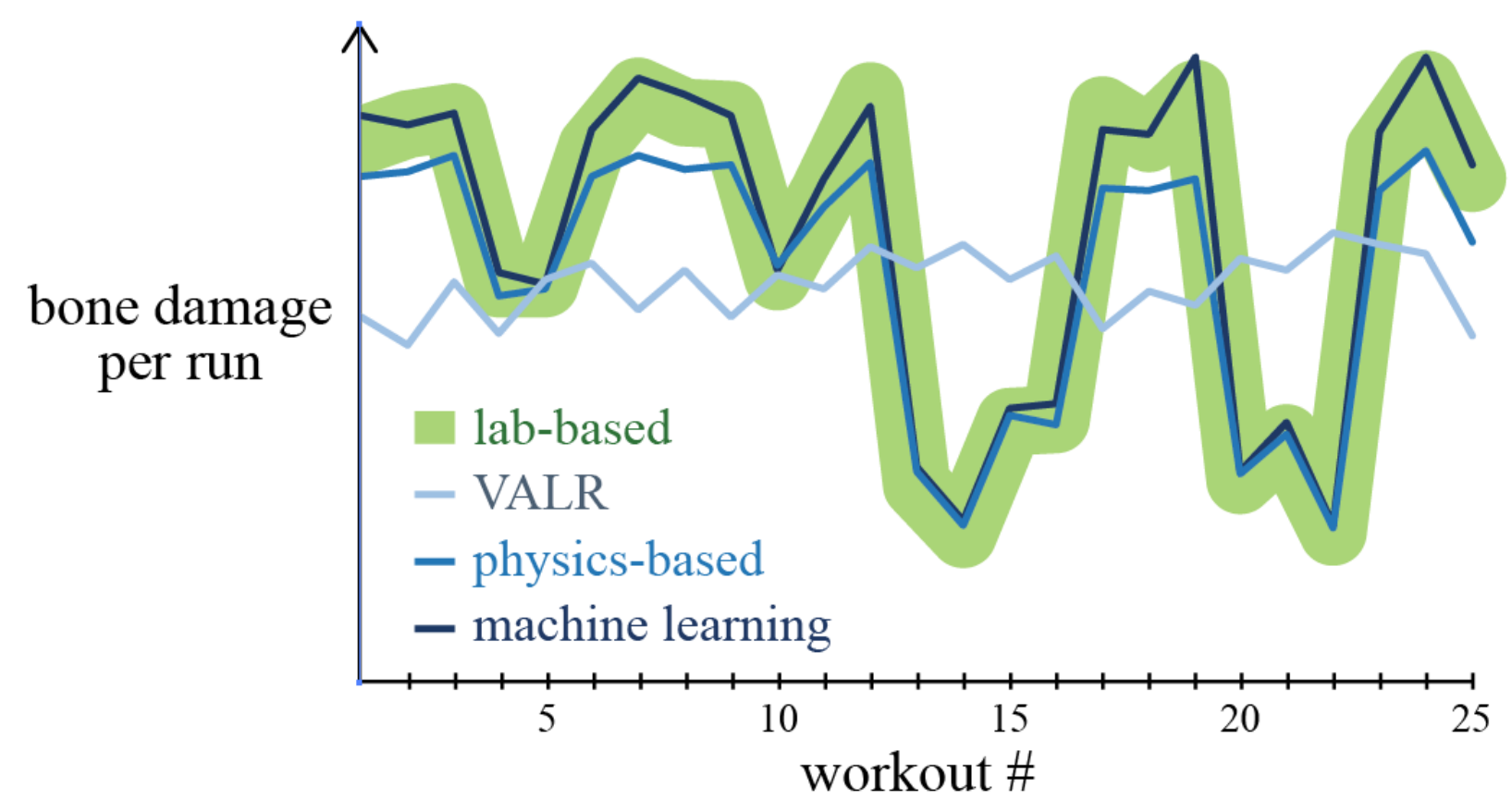

Figure 5. Example of how each algorithm performs across 25 simulated running workouts of varying intensity. This plot highlights how the physics-based (medium blue line) and machine learning (dark blue line) algorithms are able to track trends in tibial bone damage per workout over multiple running workouts. The green band represents lab-based estimates of bone damage $+/$ - a small margin of error. Using VALR (light blue line) to track bone damage across multiple runs fails to provide the runner with accurate or useful information about which workouts expose them to higher vs. lower amounts of cumulative bone damage and associated injury risk.

Considering bone damage when developing musculoskeletal load monitoring tools theoretically takes us one step closer to the mechanical mechanism underlying bone overuse injuries. While it is not yet clear what level of accuracy is sufficient for different applications (e.g., longitudinal research studies, athlete injury risk monitoring), our findings highlight the importance of considering tissue damage (in this case bone damage), not only force, when developing musculoskeletal loading and injury prevention tools.

\section{Path from new tools to scientific understanding and societal impact}

There are a couple common questions regarding monitoring bone load, damage and overuse injury risk that we have received since sharing initial results and a preprint of this study, namely: How do we know that estimates of peak bone force or associated damage will be good indicators of injury risk? How do we know which specific bone loading metric(s) to track? How do we know how accurate estimates of bone loading or damage need to be? The simple answer: We don't. This would be akin to asking: Before the invention of the pulse oximeter, how did medical device developers know this tool could be used to screen neonates for Critical Congenital Heart Disease, know the precise oximetry metrics to track, and know what level of estimation accuracy of these metrics was necessary? They didn't. Often when envisioning a new screening, diagnostic or health monitoring tool, the first versions are developed based on the state of scientific and clinical evidence at that time. Pulse oximeter developers used existing 
knowledge that monitoring oxygen saturation could potentially provide useful insight about physiological function and health. After developing an oxygen saturation estimation tool, and performing a series of research studies to refine the hardware, analytics and clinical insights, device developers were then able to use this new and validated tool to monitor neonatal (and other clinical) health and improve care, resulting in a huge societal impact.

Similarly, when envisioning a new bone stress injury risk monitoring tool, we were motivated by the current state of scientific evidence on the etiology of bone stress injuries. Specifically, we were motivated by evidence that bone stress injuries are consistent with mechanical fatigue processes due to tissue damage accumulation resulting from repetitive bone loading (Edwards, 2018; Gallagher \& Schall, 2017). Interestingly, a risk assessment tool has previously been developed to estimate tissue damage to the low back due to repeated lifting, and this tool has been validated against two occupational epidemiological injury databases (i.e., shown to explain 72-95\% of the deviance in low back disorders, Gallagher et al., 2017). Thus, there is both a strong scientific motivation and risk assessment precedence (from the occupational health field) for using tissue load monitoring in the way we propose and envision.

However, currently there is a critical technical gap: we lack the ability to monitor tibial bone loading outside the lab, which inhibits the evaluation of these mechanical fatigue processes in real world environments for runners or other individuals. Development of a novel wearable bone load (and damage) monitoring tool will enable researchers and clinicians to collect longitudinal prospective data on bone loading while also tracking injury incidence. Examining these data can then allow for testing hypotheses about the role of bone loading in the development of bone stress injuries, potentially identifying new bone health and injury risk predictive metrics. Proper measurement tools must be developed and deployed before injury risk predictive metrics will be discovered and validated. Of note, bone load and damage monitoring will likely not be used in isolation. Rather, these data may be combined with other physiologically relevant information (e.g., sleep duration, diet, bone geometry, demographics, bone bending moments) to develop deeper insights into bone health and overuse injury mechanisms. Much remains unknown about which metrics are key, how to combine them to most effectively monitor bone health or injury risk, and what accuracy of each metric is needed for different applications. What is known is that developing a wearable bone load and damage monitoring tool will provide researchers and clinicians with unique new capabilities to gain a deeper scientific understanding of bone health. Expanding knowledge related to bone stress injury mechanisms and prevention has huge potential for improving societal health, happiness, and productivity. This study represents a tangible step towards this goal.

\section{Additional limitations and opportunities}

First, we focused on one specific musculoskeletal structure (the tibia), direction of loading (tibial compression), and range of conditions which were all treated with equal weight in algorithm development (treadmill running across a range of speeds and slopes). The presented algorithms were developed within these bounds, and any additional musculoskeletal structure, direction of loading, or range of conditions would necessitate additional validation studies. Nevertheless, the same analysis could be used to investigate other musculoskeletal structures (e.g., lumbar loading for individuals at risk of back overuse injury). Our outlined process can help identify candidate sensors and signals, develop algorithms, and 
begin to establish expectations for levels of accuracy that might be achievable with existing types of sensors.

Second, algorithm development required us to choose which method of estimating tibial force (and resulting damage) was considered our ground truth. As described previously (Matijevich et al., 2019), we have confidence in lab-based estimates of tibial compression force. There is less agreement in the literature about other loading directions (e.g., tibial bending, Derrick et al., 2016; Haris Phuah et al., 2010; Meardon et al., 2015; Rice et al., 2019; Sasimontonkul et al., 2007; Scott \& Winter, 1990; Yang et al., 2014). Nevertheless, there may be value to exploring algorithms for estimating these other loading directions, or for estimating damage due to the combined loading from axial compression and bending (see Appendix B Limitations for additional discussion). However, it should be emphasized that these other loading directions are also highly dependent on muscles forces, and it is again unlikely that a single variable metric (e.g., VALR) would be an accurate predictor of internal loading.

Third, these algorithms were developed using idealized wearable signals. Actual wearable sensor signals are expected to exhibit additional noise, errors, or drift, and therefore the results presented here should be interpreted as best case expectations for wearables utilizing the sensor/signal sets described here. Future studies could use actual wearable sensor signals as model inputs, or apply noise, errors, or drift to current idealized wearable signals. However, previous validation experiments have shown good agreement between pressure insoles and the vertical GRF (Koch et al., 2016) and IMU-based kinematics and lab-based motion capture kinematics (Picerno, 2017). Additionally, sensor hardware, algorithms and signal quality are constantly improving, bringing wearable sensor measurements closer to lab-based measurements.

Fourth, with advances in sensor hardware and sensor integration techniques, the potential for development and adoption of musculoskeletal load monitoring tools into daily life will also evolve. Sensor integrated clothing, footwear, insoles, textiles, etc. are becoming increasingly common and the use of multiple sensors is becoming increasingly practical. In fact, there are already multiple commercial wearables available that contain a pressure-insole and an IMU (a combination of sensors yielding the lowest bone force estimation error in this study, Table 4). The results of this study demonstrate the potential pay-offs of leveraging a multi-sensor approach. It is important to remember that there was a time when walking around with a phone in your pocket seemed almost unimaginable. But now it is routine to walk around all day with a pocket full of sensors -- smartphones that fit seamlessly into our daily lives enhance our productivity, connectedness, health monitoring, etc. in ways that were previously unthinkable. Similarly, we expect that the limiting factor of musculoskeletal load monitoring tools will not be the number or cost of sensors, or the size of batteries, but rather the limiting factor will be our imagination, our vision, and the ability to combine data science (e.g., machine learning) and domain knowledge (e.g., biomechanics) to provide actionable and scientifically validated insights that improve societal health and well-being.

Finally, we note that the analysis here was performed on stride-averaged data. However, we expect all the same conclusions to hold true for stride-by-stride analysis, since the variability within a running condition was small relative to the variability across conditions. 


\section{Conclusion}

Here we demonstrate two multi-sensor algorithms - one physics-based and one machine learning - that offer promising solutions for estimating peak tibial force with wearable sensors. We show that by harnessing signals from existing wearable sensors, and applying multi-sensor algorithms, it is feasible to drastically improve the estimation accuracy of peak tibial force (by two or four fold relative to the current state-of-the-art in commercial wearables), and associated bone damage. This study highlights the exciting potential to combine wearables, musculoskeletal biomechanics and machine learning to develop more accurate tools for monitoring musculoskeletal loading in applied situations.

\section{Competing interests}

ESM, LRS, and KEZ are co-inventors on a patent application covering the wearable monitoring methods/algorithms described here (PCT/US2019/029790).

\section{Acknowledgements}

We thank Dr. Gerasimos Bastas for manuscript feedback, Sara Tsai and Anna Wolfe for involvement in project discussions, and Lauren Branscombe for contributing to early pilot work.

\section{Funding}

We gratefully acknowledge funding from the National Institutes of Health (R01EB028105), and institutional funding from the Vanderbilt University Discovery Grant program.

\section{Appendix A}

Appendix A is intended to detail the specific methods used in the physics-based algorithm. Eq. A1 describes the lab-based estimate of tibial force $(F(t))$ as the summation of the net force on the ankle and the force from the plantarflexor calf muscles $\left(F_{m}\right)$.

$$
F(t)=|\boldsymbol{G R} \boldsymbol{F}| \cdot \cos (\beta)+F_{m}
$$

(Equation A1)

Where $\beta$ is the 3D angle between the $\boldsymbol{G} \boldsymbol{R} \boldsymbol{F}$ and long-axis of the lower leg segment, and $F_{m}$ was estimated by dividing the net sagittal plane ankle moment by the Achilles tendon moment arm (assumed constant, $5 \mathrm{~cm}$, (Matijevich et al., 2019)). From $F(t)$ we extracted $F_{\text {max }}$, the maximum tibial force during stance. Vectors are bolded, and scalars are non-bolded.

To estimate tibial force from the available idealized wearable signals $(\hat{F}(t))$ we created a modified version of Eq. A1. We approximated both the net force on the ankle and the force from plantarflexor calf muscles (Eq. A2). The net force on the ankle was estimated by projecting the normal force under the foot $\left(G R F_{\text {normal }}\right)$ onto an estimate of the long axis of the tibia. The force from the plantarflexor calf muscles was estimated by approximating the net ankle moment, then dividing by Achilles tendon moment arm length $\left(r_{A T}\right.$, assumed constant, $5 \mathrm{~cm}$, Matijevich et al., 2019). To 
approximate the net ankle moment, we computed $\mathrm{CoP}_{a p} \cdot G R F_{\text {normal }}$, where $\mathrm{CoP}_{a p}$ was the anteriorposterior center of pressure distance relative to the ankle joint position. The ankle joint position was assumed $5 \mathrm{~cm}$ anterior to the heel of the foot, based on a typical Achilles tendon moment arm length. Summing these approximations in Eq. A2 yields an unscaled estimate of tibial force $\hat{F}^{\prime}(t)$. From $\hat{F}^{\prime}(t)$ we extracted $\hat{F}_{\text {max }}^{\prime}$, the maximum unscaled tibial force during stance.

$$
\hat{F}^{\prime}(t)=G R F_{\text {normal }} \cdot \cos (\hat{\beta})+\frac{C o P_{a p} \cdot G R F_{\text {normal }}}{r_{A T}}
$$

Where $\hat{\beta}$ is the approximate angle between $G R F_{\text {normal }}$ and the long-axis of the lower leg segment. $\hat{\beta}$ is estimated as $90^{\circ}-\theta_{\text {foot }}+\theta_{\text {shank }}$, where $\theta_{\text {foot }}$ and $\theta_{\text {shank }}$ are foot and shank angles in the sagittal plane relative to the horizontal.

Of note, Eq. A2 assumes that $C o P_{a p}$ and $G R F_{\text {normal }}$ are perpendicular to each other, neglects moment and reaction force contributions from the anterior-posterior and mediolateral GRFs and is devoid of a true ankle joint center estimate. We therefore anticipated this physics-based algorithm might systematically under- or over-estimate tibial force; thus why we refer to this initial estimate as unscaled. To account for this, linear scaling coefficients were calculated using k-fold validation, then applied to arrive at a scaled estimate of peak tibial force $\left(\hat{F}_{\text {max }}\right)$. 


\section{Appendix B}

Appendix B provides extended methods and results for estimating bone damage and cumulative bone damage over simulated running sessions. We evaluated both the bone damage per running stride and the cumulative bone damage accrued over simulated five-mile running sessions, to supplement our analysis of force estimation accuracy.

\section{Methods: Bone damage per running stride}

Bone damage per running stride was estimated using an established inverse power law for bone damage $\left(D=c F_{\text {max }}{ }^{e}\right.$ and $\widehat{D}=c \widehat{F}_{\text {max }}{ }^{e}$ ) that describes the life-stress relationship of materials under cyclic loading, where $e$ is an empirically-derived exponential constant for bone of seven (Caler \& Carter, 1989; Edwards et al., 2009; Loundagin et al., 2018). Here, $c$ is set equal to unity such that our cumulative damage are dimensionless units of relative cumulative damage. A different constant $c$ and additional modeling factors would be needed to translate $D$ into physical representations of mechanical fatigue (e.g., number of cycles until bone failure or probability of failure, (Carter \& Caler, 1985; Edwards et al., 2010)). For each algorithm, we computed the MAPE between $D$ and $\widehat{D}$ across all conditions for each runner.

\section{Methods: Cumulative damage and simulated running session}

We estimated cumulative damage over a series of loading cycles (i.e., over multiple running strides). We created 1000 simulated runs that were each approximately five miles. The series of loading cycles was constructed by randomly assigning a speed-slope combination in half mile increments, constrained by speed-slope combinations we collected in our experiment (one example in Fig. B1). To generate a plausible running session, for each subsequent increment, the slope was constrained to change by either $+3^{\circ}, 0^{\circ}$, or $-3^{\circ}$, and the speed was randomly assigned. The number of steps taken at each half mile increment was estimated based on a previously reported speed-cadence relationship (Hoeger et al., 2008).

We estimated cumulative damage over each simulated run using Miner's rule of cumulative fatigue over a series of loading cycles at different peak forces (Eqs. B1-2, (Currey, 2013; Edwards, 2018)),

$$
\begin{aligned}
& \Sigma D=\sum_{i=1}^{10} n_{i} \cdot F_{\text {max }, i}^{7} \\
& \Sigma \widehat{D}=\sum_{i=1}^{10} n_{i} \cdot \hat{F}_{\text {max }, i}^{7}
\end{aligned}
$$

Where $i$ represents the half mile increment, $n_{i}$ is the number of steps taken in that half mile increment based on the speed assigned, and $F_{\max , i}$ and $\hat{F}_{\max , i}$ represent the peak force for the speed-slope combination assigned to that half mile increment (see Fig. B1 for one example). 
For each runner, the cumulative damage $\Sigma D$ vs. $\Sigma \widehat{D}$ was plotted for all 1000 simulated runs. We then computed the MAPE for each runner. Finally, we calculated the inter-runner average and range of MAPE.

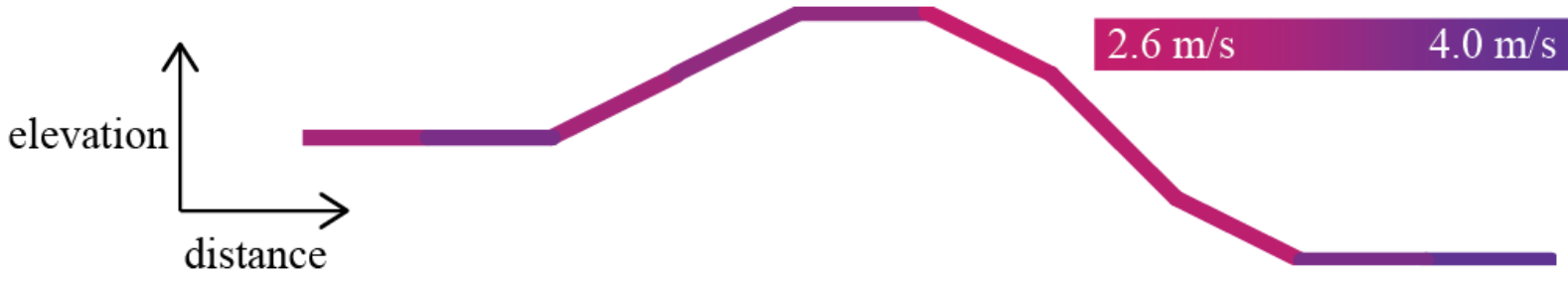

\begin{tabular}{|c|c|c|c|c|c|c|c|c|c|c|}
\hline$i$ & 1 & 2 & 3 & 4 & 5 & 6 & 7 & 8 & 9 & 10 \\
\hline slope $\left(^{\circ}\right)$ & 0 & 0 & 3 & 3 & 0 & -3 & -6 & -3 & 0 & 0 \\
\hline speed $(\mathrm{m} / \mathrm{s})$ & 3.0 & 3.4 & 3.0 & 3.2 & 3.2 & 2.6 & 2.8 & 2.8 & 3.4 & 4.0 \\
\hline$n$ (\# steps) & 750 & 650 & 750 & 750 & 750 & 850 & 850 & 850 & 650 & 550 \\
\hline$F_{\text {max }, i}(\mathrm{BW})$ & 7.9 & 8.3 & 8.3 & 8.4 & 8.0 & 7.1 & 7.3 & 7.5 & 8.3 & 8.5 \\
\hline
\end{tabular}

Figure B1. Example of one five-mile simulated running session. The embedded table shows our labbased estimates of peak tibial force $\left(F_{\max , i}\right)$ for our tenth runner. 
Results \& Discussion: Bone damage per running stride

Below are the figures and tables that support the Discussion section 4.4 Important insights gained when considering tibial damage.

A)

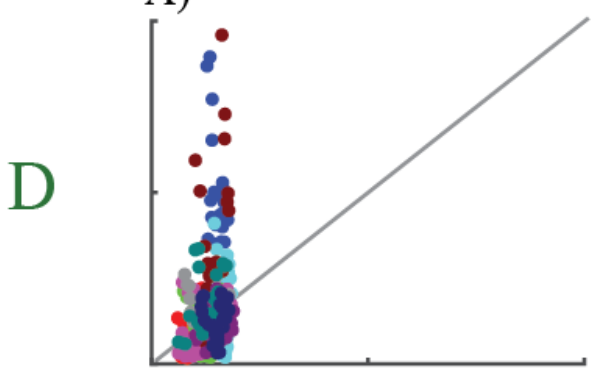

1\{
B)

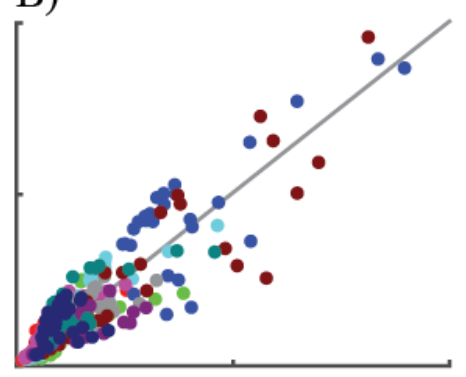

physics-based

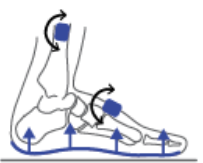

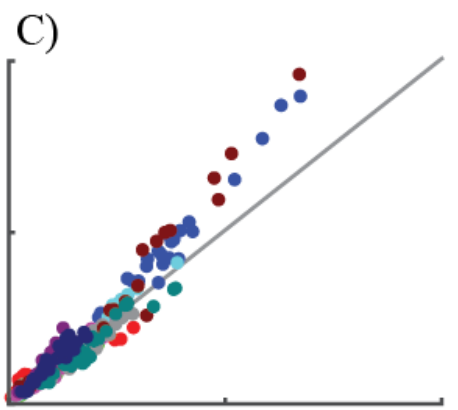

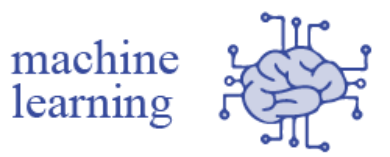

Figure B2. Comparison of bone damage based on force estimation algorithms. The plots depict bone damage estimated using lab-based peak tibial force $(D)$ vs. damage estimated using wearable sensor algorithm peak tibial force $(\widehat{D})$. Damage is reported in dimensionless units. Algorithm estimates are from A) single variable linear regression using VALR, B) physics-based algorithm, and C) machine learning algorithm. Colors represent 10 runners. Each point represents a single running speed-slope combination (30 conditions per runner). A line with a slope of one is added to visualize a perfect correspondence between lab-based and wearable estimates. See Fig. 3 for accompanying force estimation results.

Table B1. Average errors in bone damage estimates based on force estimation algorithms. The table reports MAPE of $D$ vs. $\widehat{D}$. Reported are the inter-runner means and standard deviations $(N=10)$. The physics-based algorithm and machine learning algorithm exhibited lower damage estimation errors than single variable linear regression algorithm using VALR $(\mathrm{p}=0.02$ and $\mathrm{p}<0.001$ respectively, based on Wilcoxon signed-rank test on the k-fold cross-validation error results translated into estimates of damage). See Table 2 for accompanying force estimation results.

\begin{tabular}{|l|c|c|c|}
\cline { 2 - 4 } \multicolumn{1}{c|}{} & $\begin{array}{c}\text { Single variable linear } \\
\text { regression (VALR) }\end{array}$ & Physics-based & Machine learning \\
\hline mean \pm std & $104 \pm 70 \%$ & $41 \pm 16 \%$ & $18 \pm 5 \%$ \\
\hline$[$ min max $]$ & {$[25 \% 242 \%]$} & {$[25 \% 81 \%]$} & {$[11 \% 27 \%]$} \\
\hline
\end{tabular}




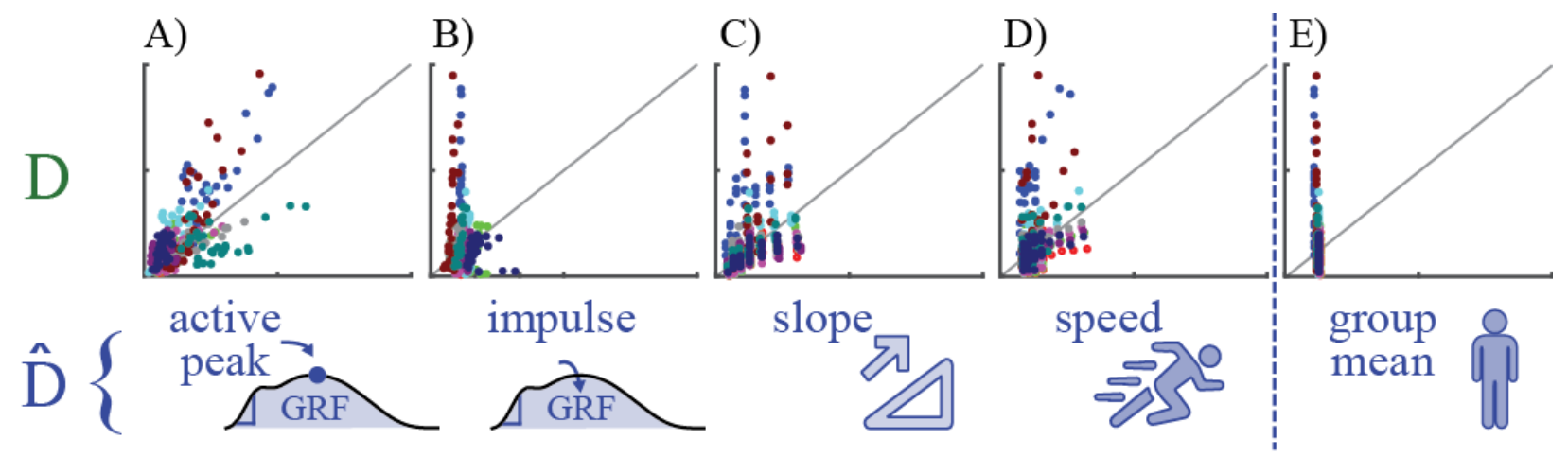

Figure B3. Comparison of bone damage based on additional single variable linear regression force estimation algorithms, and the group mean approach. The plots depict bone damage based on labbased estimates of peak tibial force $(D)$ vs. damage based on single variable linear regression estimates of peak tibial force $(\widehat{D})$. Damage is reported in dimensionless units. Single variable linear regression results used A) vertical GRF active peak, B) vertical GRF total impulse, C) ground slope, and D) running speed. Damage estimates based on E) the group mean approach are also plotted in for reference. Colors represent 10 runners. Each point represents a single running speed-slope combination (30 conditions per runner). A line with a slope of one is added to visualize a perfect correspondence between lab-based and wearable estimates. See Fig. 4 for accompanying force estimation results.

Table B2. Errors in bone damage estimates based on additional single variable linear regression force estimation algorithms, and the group mean approach. The table reports MAPE of $D$ vs. $\widehat{D}$. Reported are the inter-runner means and standard deviations $(N=10)$. See Table 3 for accompanying force estimation results.

\begin{tabular}{|l|c|c|c|c|c|}
\cline { 2 - 5 } \multicolumn{1}{c|}{} & \multicolumn{3}{c|}{ Single variable linear regression } & \multirow{2}{*}{$\begin{array}{c}\text { Group mean } \\
\text { force }\end{array}$} \\
\cline { 2 - 6 } \multicolumn{1}{c|}{$\begin{array}{c}\text { Vertical GRF } \\
\text { active peak }\end{array}$} & $\begin{array}{c}\text { Vertical GRF } \\
\text { impulse }\end{array}$ & Slope & Speed & $121 \pm 84 \%$ \\
\hline mean \pm std & $73 \pm 36 \%$ & $127 \pm 93 \%$ & $71 \pm 35 \%$ & $103 \pm 73 \%$ & {$[38 \% 261 \%]$} \\
\hline$[$ min max $]$ & {$[39 \% 132 \%]$} & {$[33 \% 304 \%]$} & {$[24 \% 133 \%]$} & {$[28 \% 234 \%]$} & {$[38 \%$. } \\
\hline
\end{tabular}

Results \& Discussion: Cumulative damage over simulated running workouts

Cumulative damage calculated using the single variable linear regression that scales VALR to estimates of peak tibial force resulted in an average MAPE of $85 \pm 59 \%$ across runners (Fig. B4D, Table B3). Cumulative damage estimated using our physics-based algorithm estimates of peak tibial force resulted in a MAPE of $31 \pm 12 \%$ (Fig. B4E, Table B3). Cumulative damage estimated using our machine learning algorithm estimates of peak tibial force resulted in a MAPE of $13 \pm 5 \%$ (Fig. B4F, Table B3).

To help visualize how errors in force translate to errors in damage, we plotted force and damage results together for a single runner (Fig. B4). We first plotted lab-based estimates of peak tibial force across 30 running conditions vs. wearable sensor algorithm estimates (Fig. B4A-C). We then plotted lab- 
based estimates of cumulative damage across 1000 simulated runs vs. wearable sensor estimates (Fig. B4D-F) directly underneath for the same runner.

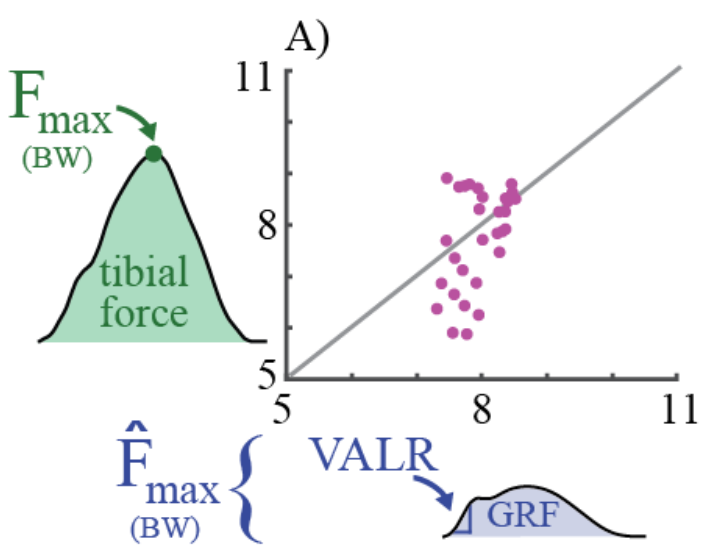

D)
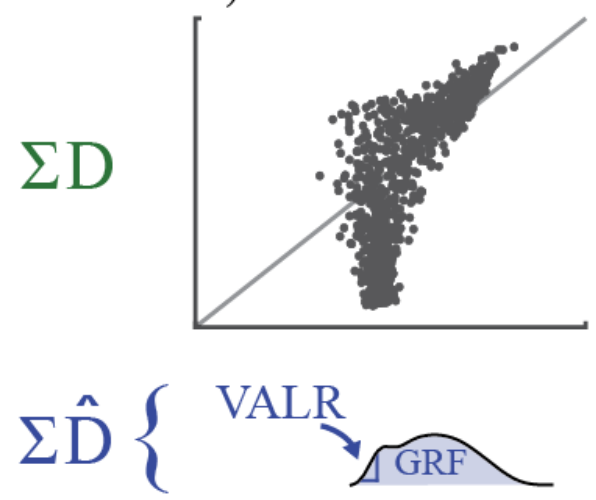

B)

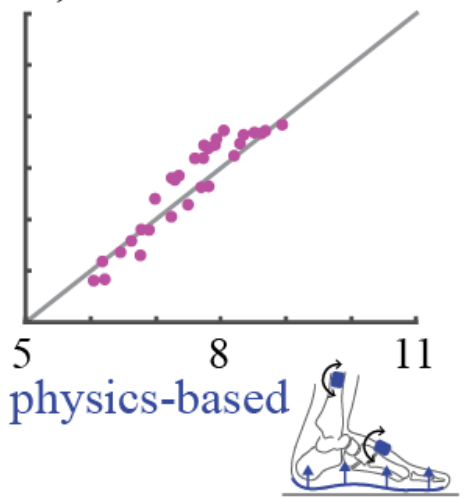

E)
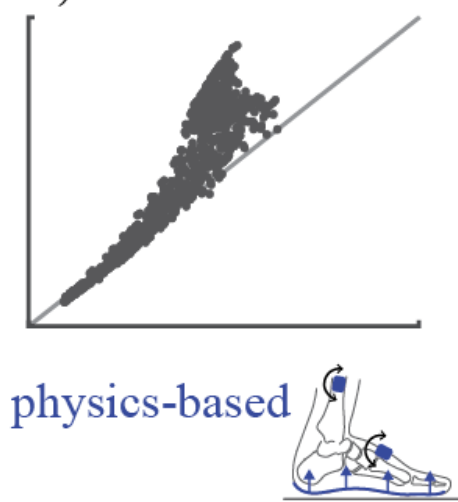

C)

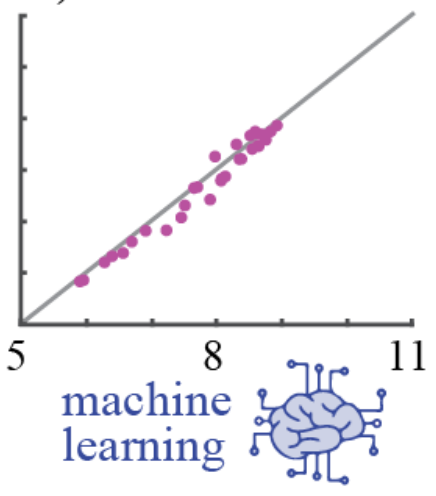

F)
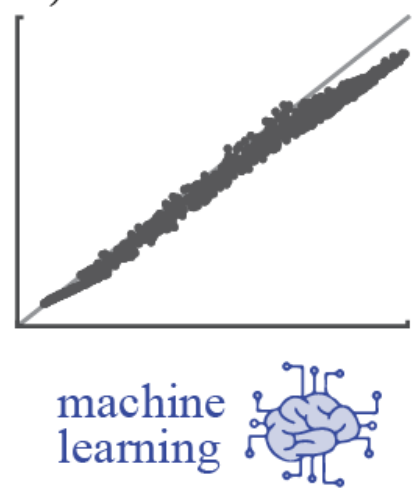

Figure B4. Cumulative damage from simulated runs for a single example runner. A-C) These plots depict lab-based estimates of peak tibial force $\left(F_{\max }\right)$ vs. wearable sensor algorithm estimates of peak tibial force $\left(\widehat{F}_{\text {max }}\right)$ for an example runner for 30 running conditions. D-F) These plots depict lab-based estimates of cumulative damage over 1000 simulated runs $\left(\sum D\right)$ vs. wearable sensor algorithm estimates of cumulative damage $\left(\sum \widehat{D}\right)$. Force is reported in units of BW and damage is reported in dimensionless units. Using VALR, a $8.5 \%$ error in tibial force translated to a $77 \%$ error in cumulative damage. Using the physics-based algorithm, a $6.8 \%$ error in force translated to a $38 \%$ error in cumulative damage. Using the machine learning algorithm, a $2.1 \%$ error in force translated to a $10 \%$ error in cumulative damage. A line with a slope of one is added to visualize a perfect correspondence between lab-based and wearable estimates.

Table B3. Average errors in relative cumulative damage from simulated runs. The table reports MAPE of $\sum D$ vs. $\sum \widehat{D}$. Reported are the inter-runner means and standard deviations $(N=10)$.

\begin{tabular}{|l|c|c|c|}
\cline { 2 - 4 } \multicolumn{1}{c|}{} & $\begin{array}{c}\text { Single variable linear } \\
\text { regression (VALR) }\end{array}$ & Physics-based & Machine learning \\
\hline mean \pm std & $85 \pm 59 \%$ & $31 \pm 12 \%$ & $13 \pm 5 \%$ \\
\hline$[$ min $\max ]$ & {$[25 \% 213 \%]$} & {$[17 \% 57 \%]$} & {$[7 \% 22 \%]$} \\
\hline
\end{tabular}


To contextualize the consequences of misestimating bone damage, we imagined a runner with a history of tibial stress fractures who wanted to monitor bone damage across multiple days of running, in order to understand which workouts lead to more or less wear and tear on her tibia. This runner would need a wearable that could be worn daily and could track bone damage for each workout, so she could evaluate when rest and recovery days are needed to help avoid a reoccurrence of the overuse injury. While this runner doesn't need a perfect estimate of bone damage, she does need the estimate to be within some reasonable margin of error. This is because if a wearable largely overestimates the cumulative damage to the bone, she may think she is at heightened injury risk, and sideline herself unnecessarily (Fig. B5). If a wearable underestimates the cumulative damage to the bone, she may not be notified of heightened injury risk in time, and fail to take rest days before an overuse injury occurs (Fig. B5).

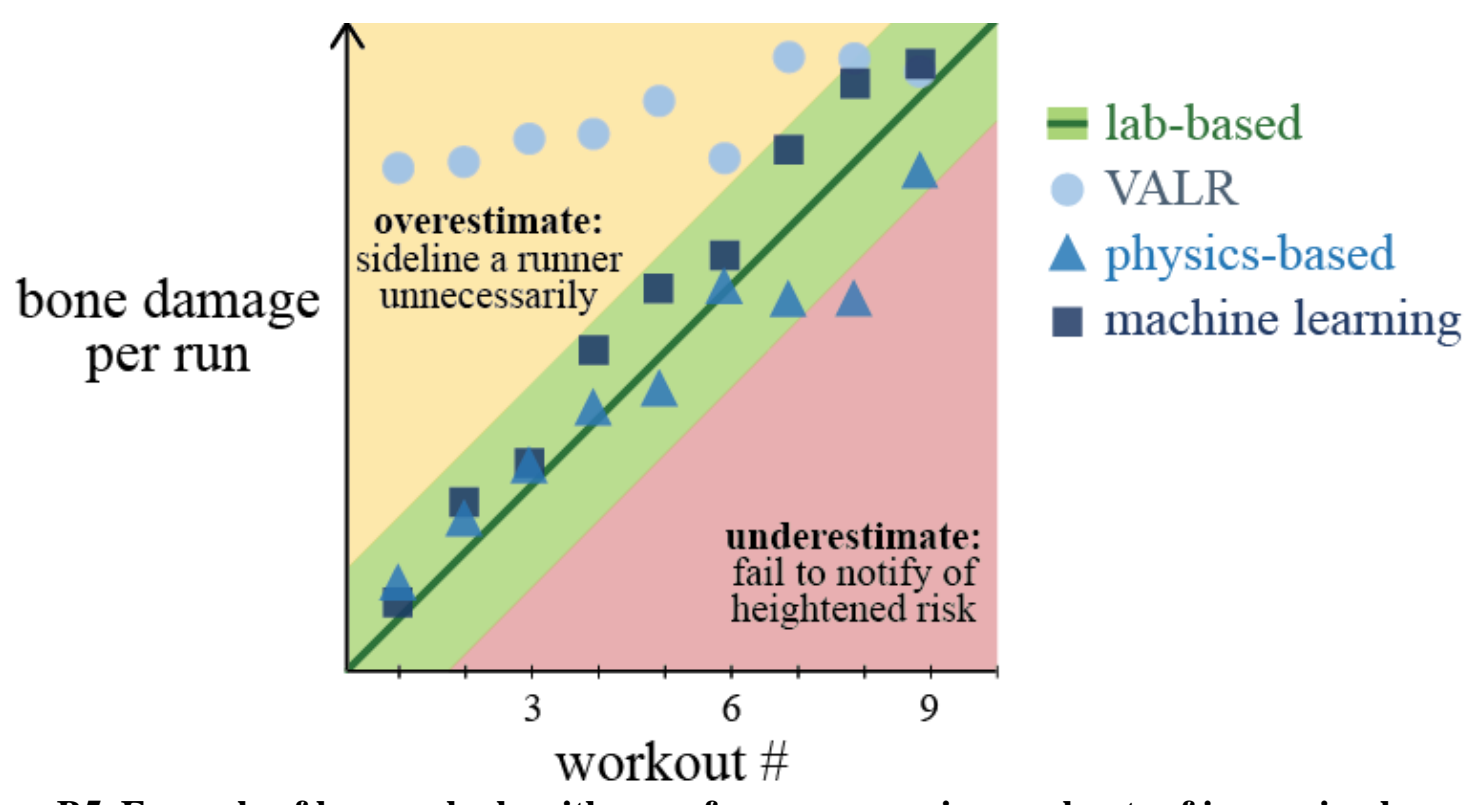

Figure B5. Example of how each algorithm performs across nine workouts of increasing bone damage. From our 1000 simulated 5-mile runs, we selected nine runs that spanned from low to high bone damage. The green line represents the lab-based estimates of bone damage per run. The green band is a visual reminder that for any application there would be some acceptable margin of error. Blue circles, triangles, and squares represent wearable estimates of bone damage using VALR, the physics-based, and the machine learning algorithms, respectively. For this subset of runs, VALR severely overestimates the damage to the bone for seven of the nine workouts. In contrast, the physics-based algorithm only underestimates the damage to the bone for one workout, and the machine learning algorithm did not yield any estimates outside the depicted margin of error.

To further illustrate how a wearable that misestimates cumulative damage may affect our imagined runner, we did the following: From our 1000 simulated five-mile running sessions, we selected a subset of 25 runs, each with a different amount of lab-based cumulative damage per run. This illustration is presented in Discussion of the main text and Fig. 5. This example also provides an important reminder that even though runs may be the same distance (5 miles), they can result in hugely different amounts of cumulative bone damage per run. 


\section{Limitations}

These cumulative damage estimates do not account for bone remodeling and adaptation (Currey, 2013) that would occur over time between these simulated workouts as our goal was only to compare damage accrued during each simulated running workout due to repeated bone loading. Interestingly, bone remodeling and adaptation are also influenced by bone load (Edwards, 2018), and therefore using estimates based on single-variable linear regression (e.g., VALR) may also limit estimation and understanding of these processes. While a wearable tool for tracking long-term bone health will likely include a composite model that combines estimates of bone damage, remodeling, adaptation, and other processes and risk factors, a reasonable estimate of bone load is foundational to estimating many of these components.

Additionally, the damage estimate here was based solely on compressive (axial) force. Future algorithms could be developed to estimate other loading directions (e.g., bending moment) and/or resulting bone stresses, and future estimates of damage may be obtained using these combined loads and/or stresses.

We note that even if we did add additional remodeling processes and/or loading directions to our estimates of damage, it would not alter the conclusions drawn here: (i) that the use of single-variable linear regression (e.g., VALR) leads to inaccurate tibial force and damage estimates, and (ii) that more accurate estimates of tibial force (e.g. using the physics-based and machine learning algorithms introduced in this study) can improve our estimates of tibial damage. 


\section{References}

Bennell, K. L., \& Brukner, P. D. (1997). Epidemiology and site specificity of stress fractures. Clinics in Sports Medicine, 16(2), 179-196. https://doi.org/10.1016/S0278-5919(05)70016-8

Brukner, P. D., Bradshaw, C., Khan, K., White, S., \& Crossley, K. M. (1996). Stress Fractures: A Review of 180 Case. Clinical Journal of Sport Medicine.

Burdett, R. G. (1982). Forces predicted at the ankle during running. Medicine and Science in Sports and Exercise, 14(4), 308-316.

Caler, W. E., \& Carter, D. R. (1989). Bone creep-fatigue damage accumulation. Journal of Biomechanics, 22(6), 625-635. https://doi.org/10.1016/0021-9290(89)90013-4

Carter, D. R., \& Caler, W. E. (1985). A cumulative damage model for bone fracture. Journal of Orthopaedic Research, 3(1), 84-90. https://doi.org/10.1002/jor.1100030110

Cosman, F., Ruffing, J., Zion, M., Uhorchak, J., Ralston, S., Tendy, S., McGuigan, F. E. A., Lindsay, R., \& Nieves, J. (2013). Determinants of stress fracture risk in United States Military Academy cadets. Bone, 55(2), 359-366. https://doi.org/10.1016/j.bone.2013.04.011

Crowell, H. P. (2010). Reducing Impact Loading During Running With the Use of Real-Time Visual Feedback. Journal of Orthopaedic \& Sports Physical Therapy, 40(4), 206-213. https://doi.org/10.2519/jospt.2010.3166

Currey, J. D. (2013). Bones: Structure and Mechanics. Princeton University Press.

Derrick, T. R., Edwards, W. B., Fellin, R. E., \& Seay, J. F. (2016). An integrative modeling approach for the efficient estimation of cross sectional tibial stresses during locomotion. Journal of Biomechanics, 49(3), 429-435. https://doi.org/10.1016/j.jbiomech.2016.01.003

Edwards, W. B. (2018). Modeling Overuse Injuries in Sport as a Mechanical Fatigue Phenomenon. Exercise and Sport Sciences Reviews. https://doi.org/10.1249/JES.0000000000000163 
Edwards, W. B., Taylor, D., Rudolphi, T., Gillette, J., \& Derrick, T. (2009). Effects of Stride Length and Running Mileage on a Probabilistic Stress Fracture Model. Medicine \& Science in Sports \& Exercise, 2177-2184. https://doi.org/10.1249/MSS.0b013e3181a984c4

Edwards, W. B., Taylor, D., Rudolphi, T. J., Gillette, J. C., \& Derrick, T. R. (2010). Effects of running speed on a probabilistic stress fracture model. Clinical Biomechanics, 25(4), 372-377. https://doi.org/10.1016/j.clinbiomech.2010.01.001

Fong, D. T.-P., Chan, Y.-Y., Hong, Y., Yung, P. S.-H., Fung, K.-Y., \& Chan, K.-M. (2008). Estimating the complete ground reaction forces with pressure insoles in walking. Journal of Biomechanics, 41(11), 2597-2601. https://doi.org/10.1016/j.jbiomech.2008.05.007

Gallagher, S., \& Schall, M. C. (2017). Musculoskeletal disorders as a fatigue failure process: evidence, implications and research needs. Ergonomics, 60(2), 255-269. https://doi.org/10.1080/00140139.2016.1208848

Gallagher, S., Sesek, R. F., Schall, M. C., \& Huangfu, R. (2017). Development and validation of an easy-touse risk assessment tool for cumulative low back loading: The Lifting Fatigue Failure Tool (LiFFT). Applied Ergonomics, 63, 142-150. https://doi.org/10.1016/j.apergo.2017.04.016

Halilaj, E., Rajagopal, A., Fiterau, M., Hicks, J. L., Hastie, T. J., \& Delp, S. L. (2018). Machine learning in human movement biomechanics: Best practices, common pitfalls, and new opportunities. Journal of Biomechanics, 81, 1-11. https://doi.org/10.1016/j.jbiomech.2018.09.009

Hamill, J., Derrick, T. R., \& Holt, K. G. (1995). Shock attenuation and stride frequency during running. Human Movement Science, 14(1), 45-60. https://doi.org/10.1016/0167-9457(95)00004-C Haris Phuah, A., Schache, A. G., Crossley, K. M., Wrigley, T. V., \& Creaby, M. W. (2010). Sagittal plane bending moments acting on the lower leg during running. Gait \& Posture, 31(2), 218-222. https://doi.org/10.1016/j.gaitpost.2009.10.009 
Hennig, E. M., Milani, T. L., \& Lafortune, M. A. (1993). Use of Ground Reaction Force Parameters in Predicting Peak Tibial Accelerations in Running. 9.

Hoeger, W. W. K., Bond, L., Ransdell, L., Shimon, J. M., \& Merugu, S. (2008). ONE-MILE STEP COUNT AT WALKING AND RUNNING SPEEDS. ACSM's Health \& Fitness Journal, 12(1), 14. https://doi.org/10.1249/01.FIT.0000298459.30006.8d

Hsiao, H., Guan, J., \& Weatherly, M. (2002). Accuracy and precision of two in-shoe pressure measurement systems. Ergonomics, 45(8), 537-555. https://doi.org/10.1080/00140130210136963

Hulkko, A., \& Orava, S. (1987). Stress Fractures in Athletes. International Journal of Sports Medicine, 08(3), 221-226. https://doi.org/10.1055/s-2008-1025659

Jacobs, D. A., \& Ferris, D. P. (2015). Estimation of ground reaction forces and ankle moment with multiple, low-cost sensors. Journal of NeuroEngineering and Rehabilitation, 12. https://doi.org/10.1186/s12984-015-0081-x

Kiernan, D., Hawkins, D. A., Manoukian, M. A. C., McKallip, M., Oelsner, L., Caskey, C. F., \& Coolbaugh, C. L. (2018). Accelerometer-based prediction of running injury in National Collegiate Athletic Association track athletes. Journal of Biomechanics, $0(0)$. https://doi.org/10.1016/j.jbiomech.2018.04.001

Koch, M., Lunde, L.-K., Ernst, M., Knardahl, S., \& Veiersted, K. B. (2016). Validity and reliability of pressure-measurement insoles for vertical ground reaction force assessment in field situations. Applied Ergonomics, 53, 44-51. https://doi.org/10.1016/j.apergo.2015.08.011

Komi, P. V. (1990). Relevance of in vivo force measurements to human biomechanics. Journal of Biomechanics, 23, 27-34. https://doi.org/10.1016/0021-9290(90)90038-5 
Lanyon, L. E., Hampson, W. G. J., Goodship, A. E., \& Shah, J. S. (1975). Bone Deformation Recorded in vivo from Strain Gauges Attached to the Human Tibial Shaft. Acta Orthopaedica Scandinavica, 46(2), 256-268. https://doi.org/10.3109/17453677508989216

Lim, S., \& D'Souza, C. (2020). A narrative review on contemporary and emerging uses of inertial sensing in occupational ergonomics. International Journal of Industrial Ergonomics, 76, 102937. https://doi.org/10.1016/j.ergon.2020.102937

Loundagin, L. L., Schmidt, T. A., \& Edwards, W. B. (2018). Mechanical Fatigue of Bovine Cortical Bone Using Ground Reaction Force Waveforms in Running. Journal of Biomechanical Engineering, 140(3), 031003-031003-031005. https://doi.org/10.1115/1.4038288

Martin, J. A., Brandon, S. C. E., Keuler, E. M., Hermus, J. R., Ehlers, A. C., Segalman, D. J., Allen, M. S., \& Thelen, D. G. (2018). Gauging force by tapping tendons. Nature Communications, 9(1), 1592. https://doi.org/10.1038/s41467-018-03797-6

Matheson, G. O., Clement, D. B., Mckenzie, D. C., Taunton, J. E., Lloyd-Smith, D. R., \& Macintyre, J. G. (1987). Stress fractures in athletes: A study of 320 cases. The American Journal of Sports Medicine, 15(1), 46-58. https://doi.org/10.1177/036354658701500107

Matijevich, E. S., Branscombe, L. M., Scott, L. R., \& Zelik, K. E. (2019). Ground reaction force metrics are not strongly correlated with tibial bone load when running across speeds and slopes: Implications for science, sport and wearable tech. PLOS ONE, 14(1), e0210000. https://doi.org/10.1371/journal.pone.0210000

Meardon, S. A., Willson, J. D., Gries, S. R., Kernozek, T. W., \& Derrick, T. R. (2015). Bone stress in runners with tibial stress fracture. Clinical Biomechanics, 30(9), 895-902. https://doi.org/10.1016/j.clinbiomech.2015.07.012 
Neugebauer, J. M., Collins, K. H., \& Hawkins, D. A. (2014). Ground Reaction Force Estimates from ActiGraph GT3X+ Hip Accelerations. PLOS ONE, 9(6), e99023. https://doi.org/10.1371/journal.pone.0099023

Patel, S., Park, H., Bonato, P., Chan, L., \& Rodgers, M. (2012). A review of wearable sensors and systems with application in rehabilitation. Journal of NeuroEngineering and Rehabilitation, 9(1), 21. https://doi.org/10.1186/1743-0003-9-21

Peake, J. M., Kerr, G., \& Sullivan, J. P. (2018). A Critical Review of Consumer Wearables, Mobile Applications, and Equipment for Providing Biofeedback, Monitoring Stress, and Sleep in Physically Active Populations. Frontiers in Physiology, 9. https://doi.org/10.3389/fphys.2018.00743

Picerno, P. (2017). 25 years of lower limb joint kinematics by using inertial and magnetic sensors: A review of methodological approaches. Gait \& Posture, 51, 239-246. https://doi.org/10.1016/j.gaitpost.2016.11.008

Ranavolo, A., Draicchio, F., Varrecchia, T., Silvetti, A., \& lavicoli, S. (2018). Wearable Monitoring Devices for Biomechanical Risk Assessment at Work: Current Status and Future Challenges-A Systematic Review. International Journal of Environmental Research and Public Health, 15(9), 2001. https://doi.org/10.3390/ijerph15092001

Rice, H., Weir, G., Trudeau, M. B., Meardon, S., Derrick, T., \& Hamill, J. (2019). Estimating Tibial Stress throughout the Duration of a Treadmill Run. Medicine \& Science in Sports \& Exercise, Publish Ahead of Print. https://doi.org/10.1249/MSS.0000000000002039

Sasimontonkul, S., Bay, B. K., \& Pavol, M. J. (2007). Bone contact forces on the distal tibia during the stance phase of running. Journal of Biomechanics, 40(15), 3503-3509. https://doi.org/10.1016/j.jbiomech.2007.05.024 
Scott, S. H., \& Winter, D. A. (1990). Internal forces at chronic running injury sites. Medicine \& Science in Sports \& Exercise, 22(3), 357-369.

Vigotsky, A., Zelik, K. E., Lake, J., \& Hinrichs, R. N. (2019). Mechanical misconceptions: Have we lost the "mechanics" in "sports biomechanics"? Journal of Biomechanics, 93, 1-5. https://doi.org/10.1016/j.jbiomech.2019.07.005

Wang, W., \& Adamczyk, P. G. (2019). Analyzing Gait in the Real World Using Wearable Movement Sensors and Frequently Repeated Movement Paths. Sensors, 19(8), 1925. https://doi.org/10.3390/s19081925

Willy, R. W. (2018). Innovations and pitfalls in the use of wearable devices in the prevention and rehabilitation of running related injuries. Physical Therapy in Sport, 29, 26-33. https://doi.org/10.1016/j.ptsp.2017.10.003

Worp, H. van der, Vrielink, J. W., \& Bredeweg, S. W. (2016). Do runners who suffer injuries have higher vertical ground reaction forces than those who remain injury-free? A systematic review and meta-analysis. Br J Sports Med, bjsports-2015-094924. https://doi.org/10.1136/bjsports-2015094924

Yagi, S., Muneta, T., \& Sekiya, I. (2013). Incidence and risk factors for medial tibial stress syndrome and tibial stress fracture in high school runners. Knee Surgery, Sports Traumatology, Arthroscopy, 21(3), 556-563. https://doi.org/10.1007/s00167-012-2160-x

Yang, P.-F., Sanno, M., Ganse, B., Koy, T., Brüggemann, G.-P., Müller, L. P., \& Rittweger, J. (2014). Torsion and Antero-Posterior Bending in the In Vivo Human Tibia Loading Regimes during Walking and Running. PLOS ONE, 9(4), e94525. https://doi.org/10.1371/journal.pone.0094525 
Zadpoor, A. A., \& Nikooyan, A. A. (2011). The relationship between lower-extremity stress fractures and the ground reaction force: A systematic review. Clinical Biomechanics, 26(1), 23-28. https://doi.org/10.1016/j.clinbiomech.2010.08.005 